\title{
Diacronie
}

Studi di Storia Contemporanea

$\mathrm{N}^{\circ} 24,4 \mid 2015$

Le dittature militari: fisionomia ed eredità politica

\section{Un passato che non passa}

L'Argentina dinanzi al caso Angelelli

Mirko Giancola

\section{(2) OpenEdition}

Journals

\section{Edizione digitale}

URL: http://journals.openedition.org/diacronie/3628

DOI: $10.4000 /$ diacronie.3628

ISSN: 2038-0925

Editore

Association culturelle Diacronie

Notizia bibliografica digitale

Mirko Giancola, "Un passato che non passa », Diacronie [Online], N²4, 4 | 2015, documento 2, Messo online il 29 décembre 2015, consultato il 01 mai 2019. URL : http://journals.openedition.org/

diacronie/3628; DOI : 10.4000/diacronie.3628 


\title{
Diacronie
}

N. 24 | 4|2015 Le dittature militari: fisionomia ed eredità politica

\section{2/}

\section{Un passato che non passa}

\section{L'Argentina dinanzi al caso Angelelli}

\author{
Mirko GIANCOLA*
}

Sovversivo per alcuni, martire per altri, la figura di Mons. Angelelli - vescovo cattolico assassinato dal regime militare argentino nel 1976 - ha finito per trascendere $i$ confini del mondo ecclesiastico, assurgendo a simbolo della resistenza alla dittatura militare. Archiviato inizialmente come incidente, il "caso Angelelli" finirà sepolto sotto la coltre di oblio sulla quale muoveva i suoi primi passi la fragile democrazia argentina, arrivando a conclusione solo trent'anni più tardi. L'obiettivo del presente articolo è di utilizzare la vicenda di Mons. Angelelli come strumento di lettura tanto delle complesse relazioni che si stabilirono tra la Chiesa argentina e il regime militare, quanto del controverso tema dei procedimenti giudiziari contro i crimini commessi durante la dittatura.

\section{Introduzione}

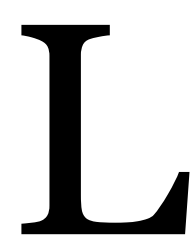

a figura di Mons. Angelelli, la sua vita come la sua morte, s'inserisce nelle complesse dinamiche che caratterizzarono le relazioni tra la Chiesa cattolica e il mondo politico e sociale argentino nella seconda metà del Novecento. Sul ruolo della Chiesa argentina nel corso dell'ultima dittatura militare si è detto e scritto molto. L'elezione al soglio pontificio di un cardinale argentino ha contribuito a riaccendere le polemiche sul tema ${ }^{1}$. Partendo da una condizione di estrema debolezza,

${ }^{1}$ CALAPA, Giampiero, «Papa Francesco, l'accusa di Verbitsky: Ho i documenti: collaborò con i dittatori», in Il Fatto Quotidiano.it, URL: < http://www.ilfattoquotidiano.it/2013/o3/15/papafrancesco-laccusa-di-verbitsky-documenti-e-testimoni-collaboro-con-dittatori/531362/ [consultato il 13 agosto 2015]. 
la Chiesa cattolica argentina consolidò la sua presenza all'interno della società nel corso degli anni Trenta del secolo scorso, periodo in cui si gettarono le basi tanto di un robusto complesso dottrinario, quanto di una solida organizzazione istituzionale². Diversi studi fanno risalire allo stesso periodo l'inizio del protagonismo delle Forze Armate nella vita politica del paese3. Di fatto i due fenomeni sono strettamente legati, situandosi al centro delle dinamiche sociopolitiche che caratterizzano l'ingresso dell'Argentina nella società contemporanea. Alcune ricerche hanno mostrato come la stretta relazione esistente tra le istituzioni ecclesiastiche e quelle militari trovasse la sua origine nella rivendicazione da parte di entrambe di un ruolo tutelare dell' identità nazionale argentina 4 . La progressiva identificazione tra i concetti di fede e cittadinanza si accompagnava tuttavia al tentativo di monopolizzare tale identità da parte di diversi settori all'interno della società. Ciò portò da un lato all'affermarsi di progetti politici totalizzanti, caratterizzati da una dinamica escludente incapace di assorbire la normale dialettica politica all'interno di un solido meccanismo democratico; dall'altro rese sempre più sottili i confini tra conflitto intra-ecclesiastico e conflitto civile.

È così che le discussioni sorte all'interno del cattolicesimo all'inizio degli anni Sessanta trascesero ben presto i confini del mondo cattolico invadendo l'arena politica. Di fatto, l'aggiornamento promosso dal Concilio Vaticano II non faceva che portare a galla le tensioni già presenti nel mondo cattolico argentino, incapace di trovare una sintesi tra le diverse correnti presenti al suo interno. Ciò che tuttavia costituisce l'elemento di maggiore interesse nel caso argentino è che la Chiesa cattolica e le Forze Armate - affermatesi come le sole istituzioni in grado di conferire legittimazione politica ai diversi attori sociali - gestivano di fatto un potere di inclusione/esclusione all'interno della comunità politica. Detto in altre parole, l'esclusione di alcuni settori della società dai confini dell'ortodossia cattolica equivaleva per alcuni - soprattutto per i militari - alla loro esclusione dai confini della patria, dal cosiddetto "ser nacional". L'attacco rivolto da tali gruppi all'unità della Chiesa in un contesto di forte conflittualità politica e sociale equivaleva dunque ad un attacco all'unità della nazione; essi apparivano come infiltrati, nemici interni della patria, che le Forze Armate avevano il compito di proteggere e tutelare. ${ }^{5}$ In tale contesto, alcuni decisero di portare avanti il

2 MALLIMACI, Fortunato, Catholicisme et État militaire en Argentine 1930-1946, Tesi di Dottorato in Sociologia, Ecole des Hautes Etudes en Sciences Sociales, Paris, 1988.

3 ROUQUIE, Alain, Pouvoir Militaire et Société Politique En République Argentine, Paris, Presses de la Fondation nationale des sciences politiques, 1978.

4 ZANATTA, Loris, Del Estado Liberal a la Nación Católica. Iglesia y Ejercito en los orígenes del Peronismo 1930-1943, Buenos Aires, Universidad Nacional de Quilmes, 1996.

5 ZANATTA, Loris, «El precio de la Nación Católica. El Vaticano y el golpe de estado de 1976», in Puentes, 23, 2008, pp. 83-98. 
proprio impegno ideale entrando a far parte delle organizzazioni armate clandestine, scontrandosi con le Forze Armate e con le formazioni paramilitari formatesi in ambienti reazionari, all'interno dei quali militavano altrettanti cattolici di segno opposto. Possiamo dunque concordare con le parole di Zanatta quando afferma che, in un tale stato di cose:

Qualsiasi interpretazione dell'attitudine ecclesiastica dinanzi al regime sorto il 24 Marzo 1976 che si limiti a registrare l'intensità della condanna o del sostegno, sarebbe futile e superficiale. La storia e le caratteristiche del cattolicesimo argentino, così come la natura del suo rapporto con le Forze Armate, obbligano a considerare la Chiesa non come un arbitro del corso politico che si aprì, ma [...] come parte di esso ${ }^{6}$.

In questo articolo focalizzeremo la nostra attenzione sulla figura di Mons. Enrique Angel Angelelli, assassinato nell'agosto 1976 per volontà delle autorità militari alla guida del paese. Lungi dall'avere intenzioni agiografiche, tale articolo si propone di ricostruire la vita di Mons. Angelelli situandola all'interno delle dinamiche che caratterizzavano il rapporto della Chiesa cattolica con il regime militare che nel Marzo 1976 inaugurò il Proceso de Reorganizacion Nacional (PRN). Al racconto dei primi anni di attività del vescovo seguirà la ricostruzione della catena di eventi che lo portò a scontrarsi tanto con i gruppi di potere presenti nella sua diocesi, quanto con le autorità militari sia a livello locale che nazionale. Nell'ultima parte si proporrà infine una ricostruzione della vicenda processuale iniziata il giorno successivo alla morte del vescovo e conclusasi trenta anni dopo con la condanna del Generale Luciano Benjamin Menéndez e del Vice-ammiraglio Luis Fernando Estrella come mandanti dell'omicidio.

\section{Un riojano más: la pastorale popolare di Mons. Angelelli dal Cordobazo al Proceso de Reorganización Nacional}

Mons. Angelelli assume l'incarico presso la diocesi de la Rioja il 24 Agosto 1968, lo stesso giorno in cui a Medellín, in Colombia, i vescovi latinoamericani si riunivano per studiare l'applicazione dei dettami conciliari al continente sudamericano. Nel paese si viveva intanto un clima di protesta sociale contro le politiche economiche del regime militare installatosi nel 1966 e che, proprio a causa dei disordini verificatisi nel 1969 a

${ }^{6}$ ZANATTA, Loris, «El precio de la Nación Católica. El Vaticano y el golpe de estado de 1976», cit., p. 87. 


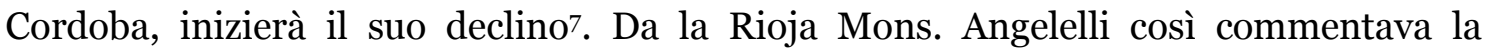
situazione nazionale: «El grito de rebeldía lanzado por la juventud y por la clase obrera [...] debe ser leído e interpretado proféticamente, se nos acaba de decir [...] que es necesario cambiar de rumbo [...] Asumamos este grito en lo que tiene de verdadero, auténtico, dramático» ${ }^{8}$. Pochi mesi più tardi il vescovo mostrava d'altra parte di essere ben conscio delle fratture che percorrevano il cattolicesimo argentino: «Unos miran a la Iglesia esperanzados y apasionadamente, descubriendo en ella la luz y la actitud abierta de servidora para todos... Otros la miran con desconfianza o la rechazan... la quieren descomprometida con la suerte de nuestro pueblo y solamente guardiana del templo» 9 .

Alle tensioni con il governo si accompagnavano quelle con alcuni settori del laicato i quali, colpiti nei propri interessi dalla pastorale diocesana, denunciavano un'eccessiva attenzione all'ambito materiale a discapito della dimensione spirituale. Ciò che precipitò lo scontro tra il vescovo e i potentati locali fu infatti il sostegno fornito dalla diocesi ad una serie di movimenti di tipo cooperativo, tra i quali il Movimiento Rural Diocesano e la Cooperativa de Trabajo Amingueños Limitada (CODETRAL). Tali organizzazioni reclamavano l'espropriazione di terreni incolti e la loro distribuzione alla CODETRAL, attaccando in tal modo posizioni di potere che reagirono con violenza.

L'apice dello scontro tra Mons. Angelelli e i suoi oppositori si raggiunse nel Giugno del 1973, quando il vescovo si recò con alcuni suoi collaboratori nella località di Anillaco, in occasione delle celebrazioni per la festa patronale. Saputo del suo arrivo, un gruppo di oppositori raccoltisi in un'organizzazione auto-denominata Cruzados de la F" organizzò una manifestazione, reclamando l'allontanamento di Mons. Angelelli, il quale per evitare ulteriori problemi decise di abbandonare la manifestazione, scomunicando però gli organizzatori della protesta. Il livello della tensione raggiunto nella diocesi spinse le autorità vaticane ad intervenire direttamente. Mons. Vicente Zazpe, arcivescovo di Santa Fé, fu nominato rappresentate del Papa per dirimere il contenzioso tra la curia diocesana e i suoi oppositori. Al termine della sua visita nella diocesi de la Rioja, Mons. Zazpe inviò una relazione alla Segreteria di Stato vaticana nella quale si riconosceva la strumentalizzazione di argomentazioni religiose da parte di settori intenti a portare avanti un conflitto di natura socio-economica, confermando un

\footnotetext{
7 Si veda: O’DONNELL, Guillermo A., El Estado burocrático autoritario, 1966-1973. Triunfos, derrotas y crisis, Buenos Aires, Prometeo libros, 2009.

8 BARONETTO, Luis, Vida y martirio de Mons. Angelelli. Obispo de la Iglesia Catolica, Cordoba, Tiempo Latinoamericano, 2006, p. 74.

9 Ibidem.
} 
certo isolamento della diocesi Riojana dal resto dell'episcopato, così come lamentato da numerosi sacerdoti ${ }^{10}$.

Intanto la repressione proseguiva nel febbraio 1976 con l'arresto del vicario diocesano di La Rioja Mons. Esteban Inestal, il quale successivamente avrebbe raccontato come, durante gli interrogatori, i militari accusassero Giovanni XXIII e Paolo VI di distruggere la Chiesa di Pio XII; dichiarando che la Chiesa di la Rioja era separata dalla chiesa argentina. Allarmato dal crescere della repressione Mons. Angelelli scriveva il 25 febbraio alle autorità episcopali argentine:

Urge una evaluación más profunda... Necesitamos urgentemente clarificar la misión que nos corresponde a las Diócesis y a la Vicaria Castrense...Es hora que abramos los ojos y no dejemos que Generales del Ejército usurpen la misión de velar por la Fe Católica...No es casualidad querer contraponer la Iglesia de Pío XII a la de Juan y Pablo... Hoy cae un Vicario General; mañana (muy próximo) caerá un Obispo. ${ }^{11}$

Nella sua risposta il vicepresidente della CEA Mons. Zazpe riferiva di aver esposto i fatti da lui denunciati al Gen. Videla, che si era impegnato a prendere provvedimenti in merito. ${ }^{12}$ Tuttavia, malgrado le rassicurazioni di Mons. Zazpe, la maggioranza dell'Episcopato -terrorizzato dalle divisioni che laceravano il cattolicesimo argentino concentrò i propri sforzi nella ricomposizione del mondo cattolico, accogliendo con favore l'avvento di un regime che prometteva di ristabilire l'ordine dentro e fuori dal tempio. Speranza e fiducia provenivano anche dalla Santa Sede, rassicurata tanto dall'opinione favorevole dell'Episcopato nazionale, quanto dal tradizionale sentimento cattolico delle Forze Armate argentine. ${ }^{13}$ La tensione crebbe tuttavia quando la repressione iniziò ad abbattersi sul clero socialmente più avanzato.

Con il golpe del 24 marzo 1976 infatti, la repressione si fece ancora più pesante, assumendo nella provincia de La Rioja i toni di una "caccia alle streghe". ${ }^{14}$ A fine aprile Mons. Angelelli scriveva al Vicepresidente della CEA denunciando come lo stesso fronte oppositore formato dai grandi proprietari della La Rioja fosse divenuto il mentore delle autorità militari, confezionando liste di sospetti affinché fossero arrestati e chiudeva la

10 TRIBUNAL ORAL FEDERAL DE LA RIOJA, Causa de lesa Humanidad "MONSEÑOR ANGELELLI", La Rioja, Poder Judicial de la Nación, 2014, p. 431.

${ }^{11}$ BARONETTO, Luis, op. cit., p. 180.

12 TRIBUNAL ORAL FEDERAL DE LA RIOJA, op. cit. p. 284.

${ }^{13}$ ZANATTA, Loris, « El precio de la Nación Católica. El Vaticano y el golpe de estado de 1976 », cit., p. 88.

14 BARONETTO, Luis, op. cit. p. 185. 
missiva chiedendo l'appoggio della Conferenza Episcopale ${ }^{15}$. Di fatto, malgrado la loro profonda aderenza ai valori del cattolicesimo, o forse proprio in virtù di essi, i militari mal sopportavano la pastorale promossa dal vescovo riojano; insofferenza acuita dall'alto grado di pubblicità che essa raggiunse. Ogni atto vessatorio da parte dei militari era comunicato da Angelelli ai suoi fedeli così come alle autorità ecclesiastiche argentine e vaticane. L'irritazione dei militari per tali denuncie traspare dai loro commenti, come quello del Vice-ammiraglio Luis Fernando Estrella, che durante una cerimonia presso il CELPA nel maggio 1976 affermava: «[...] Debemos adherir al occidentalismo, pero siempre que ese occidentalismo cristiano sea verdaderamente cristiano, como Cristo quiere al mundo, no como el hombre disfrazado de oveja quiere que sea Cristo» ${ }^{16}$. O come il discorso tenuto dal Comandante Osvaldo Héctor Pérez Bataglia nel luglio 1976, presso la Guarnigione militare di La Rioja:

La ciudadanía toda debe combatir delincuentes que disfrazados de soldados, destruyen la vida de los defensores de nuestra nacionalidad; al que caracterizado de representante de Dios, ora por la integridad o el descanso de los traidores a la patria; al que mimetizado de maestro, trastoca con su enseñanza los valores espirituales, el estilo de vida y los grandes fines morales de nuestro pueblo. ${ }^{17}$

Dinanzi al procedere delle intimidazioni Mons. Angelelli decise di sospendere le celebrazioni religiose presso la cappella del Centro de Ensayo y Lanzamiento de Proyectiles Autopropulsados (CELPA), centro dell'Aviazione situato nella località di Chamical. Tale misura fu duramente attaccata dagli oppositori del vescovo raccolti intorno al quotidiano «El Sol» che titolava «Angelelli celebra la messa per i Montoneros e nega assistenza spirituale ai servitori della Patria». ${ }^{18}$ Nel frattempo il Pro-vicario castrense Mons. Victorio Bonamin arrivava in soccorso ai militari scavalcando l'autorità del vescovo diocesano. La figura di Mons. Bonamin è centrale per comprendere le tensioni che attraversavano la Chiesa nelle sue relazioni con le Forze Armate. Il sacerdote Arturo Pinto racconta al processo di essere stato destinato come sacerdote presso il CELPA ma di aver incontrato qui la resistenza dei militari, i quali pretendevano di determinare l'orientamento pastorale della catechesi all'interno

15 BARONETTO, Luis, op. cit., p. 188.

16 TRIBUNAL ORAL FEDERAL DE LA RIOJA, op. cit., p.355.

17 Ibidem.

18 BARONETTO, Luis, op. cit., p. 183. 
dell'istituto. Rifiutandosi Pinto di acconsentire a tale richiesta, non fu più chiamato dai militari ${ }^{19}$. Di fatti il 27 giugno Mons. Bonamin annota sul suo diario:

En Chamical CELPA cumple 15 años y juran conscriptos de la clase. No rechacé invitación en vista de la triste situación espiritual de nuestra gente, sobre la cual pesa la pena de "entredicho" - práctico - sancionado por el Obispo diocesano. Acudir a la base para oficiar misa o predicar. Él quiere que vaya el párroco del lugar, que es rechazado por la base ${ }^{20}$.

Nella sua omelia al CELPA inoltre, Mons. Bonamin affermava:

La guerrilla, que mueve el país por los hijos degenerados de la Argentina contra su propia nación, o se creen el diablo y entonces interpretan los hechos, o no se creen y uno se encuentra delante del misterio de la iniquidad del que habla la misma Sagrada Escritura. Carecéis [riferendosi ai militari] de una asistencia espiritual, a la que tenéis derecho ${ }^{21}$.

\section{Para que todos sean uno: il progressivo isolamento di Mons. Angelelli}

Le preoccupazioni già da tempo segnalate da Mons. Angelelli alle autorità ecclesiastiche furono esposte in dettaglio a metà Maggio in occasione di un'Assemblea Straordinaria dell'Episcopato, ma ancora una volta il suo appello non servì a provocare una presa di posizione pubblica in sua difesa da parte del resto del corpo episcopale. Nella carta inviata a Mons. Zazpe il 13 luglio 1976, Angelelli scrive:

Te debo confesar que me hizo mucho mal la participación en la última asamblea, repito que no les pido nada; solo la elemental comunión episcopal cuando lo que está en juego es nuestra misión episcopal y, en definitiva, la Iglesia. Les repito que me digan si se tomará alguna medida desde arriba; de lo contrario buscaré otro camino. No pienso sólo en La Rioja, sino en toda la Argentina. Se me ha quitado la radio y diarios locales para poderme comunicar con mi pueblo. Lo que no entiendo es que éstos se usen por vía eclesiástica castrense para orientar a la diócesis, en el que determina el modo y el contenido sea el jefe militar; los obispos, ¿̇para qué estamos $^{22}$ ?

19TRIBUNAL ORAL FEDERAL DE LA RIOJA, op. cit., p. 393.

${ }^{20}$ Ibidem, p. 390.

${ }^{21}$ Ibidem.

${ }^{22}$ Ibidem, p. 288. 
La lettera prosegue riferendo di un'incontro con il Gen. Menéndez a Buenos Aires. Mons Angelelli si dice preoccupato dal fanatismo mostrato dal generale, e lamenta l'intromissione dei militari nelle questioni religiose:

\begin{abstract}
Sus palabras textuales nos deben hacer pensar mucho: "Mons. Mi sueño es poder recibir de mi obispo diocesano, la investidura de cruzado de la Fe y poder empuñar en una mano una espada y en otra la cruz de Cristo para eliminar los enemigos de Dios y de la Patria". El "tercer mundo", según ellos, es lo que ha dividido la Iglesia Argentina. Los interrogatorios a todos los detenidos siguen siendo en base a la relación con la Iglesia y con el Obispo. Creo que es necesario que clarifiquemos a tiempo la "usurpación" que los militares están haciendo en materia de "Fe", en regular la "misión" de la Iglesia²3.
\end{abstract}

Tale atteggiamento da parte dei militari sembra contare, tra l'altro, con l'appoggio del Vicariato Castrense, di cui Angelelli denuncia l'intromissione nella gestione della pastorale diocesana.

Mons. Bonamín vino a La Rioja y le faltó sólo nombrarme en su "arenga" en CELPA. El Nuncio está informado. Clarifiquemos criterios pastorales en este sentido. No es posible que un capellán militar sea como el "obispo" local y pueda tener él las garantías para celebrar y regular la liturgia, según disposición del jefe militar; con, por lo menos, el silencio de la V. Castrense ${ }^{24}$.

Nel mese di luglio 1976 si assiste ad un'escalation repressiva nei confronti del clero progressista. Il 5 luglio in una parrocchia di Buenos Aires venivano assassinati tre sacerdoti e due seminaristi appartenenti all'ordine dei Padri Pallottini. Due settimane dopo i sacerdoti Gabriel Longueville e Carlos de Dios Murias furono sequestrati nella località di Chamical; i loro corpi furono trovati due giorni più tardi, crivellati di proiettili e con evidenti segni di tortura. Il 23 luglio seguiva infine l'assassinio di un attivista laico del Movimiento Rural, Wenceslao Pedernera. A Roma, benché le autorità vaticane iniziassero a mostrare un certo disagio nei confronti della politica repressiva del regime, non si superò il confine di una formale protesta presso l'ambasciata Argentina. Tra gli elementi che impedirono alla Santa Sede l'assunzione di una posizione più decisa nei confronti del regime vi fu probabilmente l'attitudine benevola di gran parte dell'episcopato argentino nei confronti dei militari. Lo stupore del

\title{
23 Ibídem.
}

24Ibídem. 
Sottosegretario di Stato vaticano Benelli per il fatto che in un paese cattolico si assassinassero membri del clero ${ }^{25}$ evidenziava tuttavia l'incomprensione da parte delle autorità vaticane del ruolo centrale attribuito dai militari argentini al fattore religioso.

Il documento denominato Planes del Ejército contribuyentes al Plan de Seguridad Nacional descrive la pianificazione del colpo di Stato con le relative strategie di esecuzione. Nel Anexo II si determinano le caratteristiche di quelli che erano considerati come nemici del PRN, individuati all'interno di diverse organizzazioni sociali. Per quanto riguarda le organizzazioni religiose si cita esplicitamente il MSTM del quale si scrive:

De definida prédica socializante sirve, a la postre, a la lucha de clases que pregona el marxismo. La representación de este movimiento se materializa casi exclusivamente en los denominados Sacerdotes del Tercer Mundo, quienes en posturas contra el nuevo gobierno, serían los particulares responsables ${ }^{26}$.

L'attenzione rivolta dai militari al fattore religioso appare evidente poi dalla documentazione custodita negli archivi della Dirección de Inteligencia de la Policía de la Provincia de Buenos Aires (DIPBA). Di estremo interesse un documento del luglio 1977, intitolato Tendencias de Obispos, Arzobispos y Auxiliares, nel quale si classificano i membri dell'Episcopato argentino a seconda della loro attitudine nei confronti del PRN, nonché su base dottrinaria, dividendo il corpo episcopale in Conservatori, Moderati, Progressisti e Progressisti Avanzati, includendo in quest'ultima categoria:

Aquéllos que asumen en lo político marcadas referencias al populismo y a determinadas formas de socialismo, y que en nuestro país, no llega a configurar una tendencia marxista como ocurre en otras naciones de Latinoamérica. Ponen en su acción pastoral especial énfasis en la promoción humana de los más necesitados y en la condena taxativa a todas las formas de injusticia. Antiautoritaristas y, por ende, de alguna manera antimilitaristas ${ }^{27}$.

Essendo deceduto al momento dell'elaborazione del documento, Mons. Angelelli non compare nella classificazione della DIPBA. Vi è tuttavia un fascicolo personale su

${ }_{25}$ ZANATTA, Loris, «El precio de la Nación Católica. El Vaticano y el golpe de estado de 1976», cit., p. 92.

26 TRIBUNAL ORAL FEDERAL DE LA RIOJA, op. cit., p. 409.

${ }^{27}$ Achivio della Direccion de Inteligencia de la Policia de Buenos Aires (DIPBA), Mesa D «e», Factor Religioso, Legajo 651. La Plata. 
di lui all'interno dell'archivio della Polizia provinciale de La Rioja, redatto nell'aprile 1973, nel quale si dice a proposito del vescovo: «ideología: tercermundista marxista; anotación: "Máximo dirigente tercermundista en nuestro medio"»28.

Consapevole del suo progressivo isolamento, il prelato scriveva una lunga lettera il 5 luglio 1976 al Nunzio apostolico Pio Laghi, raccontando il clima di persecuzione vissuto dalla diocesi riojana:

El Jefe Militar OSVALDO PEREZ BATAGLIA ha venido tomando las siguientes determinaciones: quitar la Misa radial del Obispo de la Catedral y hacerla celebrar en el Casino de Suboficiales dentro del Cuartel por el Capellán Militar Felipe Pelanda López - de esto comuniqué a la Asamblea y a Mons. Tortolo. Recogí silencio... [...] A todos los presos se le hacen preguntas fundamentales acerca de la relación con el Obispo, sacerdotes, religiosas e instituciones de la Iglesia [...] Estamos permanentemente obstaculizados para cumplir con la misión de la Iglesia. Personalmente los sacerdotes y las religiosas somos humillados, requisados y allanados por la policía con orden del ejército. El jefe de Policía, Mayor Di Césaris, al "demorar" a seis religiosas entre las cuales estaba la Provincial de las Azules, Madre María Eugenia, públicamente se les dijo que eran sospechadas y que el mayor ideólogo marxista era el obispo (iridículo!), pero hasta esto llegamos. Me aconsejan que se lo diga: nuevamente he sido amenazado de muerte ${ }^{29}$.

Il 22 luglio il Nunzio risponde limitandosi a prendere conoscenza dei fatti riferiti da Mons. Angelelli e promettendo di far presente le problematiche da lui sollevate alle autorità competenti30. Nei giorni successivi Mons. Angelelli si recò nuovamente nella città di Chamical, raccogliendo ulteriori elementi sull'assassinio dei padri Longueville e Murias in un nuovo informe, nel quale appariva una nota che recitava: «Possiedo altre informazioni, altamente compromettenti, che per prudenza non posso scrivere...».31 Sulla strada del ritorno, intorno alle 14.30, l'auto in cui Mons. Angelelli viaggiava insieme al sacerdote Arturo Pinto fu spinta fuori strada provocando l' incidente in cui il vescovo perse la vita.

28 TRIBUNAL ORAL FEDERAL DE LA RIOJA, op. cit., p. 410.

29 Ibidem, pp. 531-532.

${ }^{30}$ Ibidem, p. 384.

${ }^{31}$ Ibidem, p. 533. 


\section{4. "Uno strano incidente": il caso Angelelli dalla dittatura ai giorni nostri}

Il primo processo per la morte del vescovo Angelelli si aprì il giorno successivo alla sua morte. Sin dalle prime ore le autorità militari attribuirono la causa della morte di Angelelli ad un incidente automobilistico; negli stessi termini si espressero gli organi di stampa e tale fu la versione accettata dalle stesse autorità episcopali malgrado i dubbi sollevati dal Vaticano, che sulle colonne dell'Osservatore Romano parlò di "misterioso incidente" 32 . Dopo solo venticinque giorni il giudice militare archiviava dunque il caso confermando la versione ufficiale dell'incidente automobilistico. Terminata la dittatura, alcuni esponenti dell'episcopato organizzarono una conferenza stampa in cui chiesero la riapertura del caso, apportando nuove prove a sostegno della tesi dell'omicidio. La riapertura delle indagini sulla morte di Mons. Angelelli s'inseriva nel processo di ricostruzione della democrazia argentina inaugurato con l'elezione del radicale Raul Alfonsin alla presidenza della repubblica. Nei primi mesi di governo, si procederà alla formazione della Comision Nacional sobre la Desaparicion de Personas (CONADEP), incaricata di far luce sulla politica repressiva della dittatura. La Commissione consegnò la sua relazione - intitolata Nunca Mas - nel settembre del 1984, fornendo gli strumenti che permetteranno la costruzione del Juicio a las Juntas Militares, concluso alla fine di quello stesso anno con la condanna dei massimi vertici delle Forze Armate per i crimini commessi durante la dittatura.

Diverse testimonianze di vittime della repressione nella provincia di La Rioja raccontavano di come i militari li interrogassero sulla persona di Mons. Angelelli, sui suoi legami con la CODETRAL e con i sacerdoti Murias e Longueville, lasciando intendere come l'intenzione fosse quella di raccogliere materiale in grado di dimostrare l'attitudine sovversiva del vescovo 33 Un ulteriore apporto venne poi da una cartella depositata al processo dalla diocesi de La Rioja, che Mons. Angelelli portava con se il giorno della sua morte. I documenti all'interno della cartella costituivano i risultati delle indagini effettuate da Mons. Angelelli sulla morte dei padri Longueville e Murias. Tra di essi vi era un Informe personal con la cronaca degli eventi che avevano portato alla morte dei sacerdoti. Sulla base delle nuove prove raccolte, il giudice Aldo Morales dichiarò nel giugno 1986 che «La morte di Mons. Enrique Angel Angelelli non fu dovuta ad un incidente automobilistico, ma ad un omicidio freddamente premeditato e

\footnotetext{
${ }^{2}$ L'Osservatore Romano, 22 settembre 1976, p. 5 .

33 TRIBUNAL ORAL FEDERAL DE LA RIOJA, op. cit., p. 430.
} 
atteso dalla vittima»34. Allo stesso tempo però il giudice si dichiarava incapacitato a determinare i responsabili dell'omicidio entro i termini stabiliti dalla legge per la prescrizione dei reati penali, lasciando dunque l'omicidio senza responsabili. Nei mesi successivi il procedimento penale subiva una nuova battuta d'arresto in seguito alla promulgazione delle leggi di Punto Final e di Obediencia debida. Tali leggi, varate dal governo Alfonsin in seguito ad una serie di sollevamenti militari, di fatto impedivano l'istruzione di qualsiasi procedimento penale contro i crimini commessi durante il periodo della dittatura militare. Ad esse seguirono gli indulti concessi dal Presidente Menem nel 1990 per i militari e i guerriglieri condannati nel 1985, chiudendo così definitivamente il tema della responsabilità penale per i crimini commessi durante la dittatura.

Tali provvedimenti rimarranno in vigore fino al 2005, quando il nuovo governo guidato dal presidente Nestor Kirchner decise di abolirle, spianando così la strada alla riapertura dei processi. Negli stessi anni, anche all'interno dell'episcopato argentino iniziava ad affermarsi un'attitudine maggiormente collaborativa nei confronti della giustizia civile. Costantemente attestata su posizioni di estrema cautela, l'episcopato argentino aveva infatti sino ad allora evitato di lasciarsi coinvolgere nella vicenda giudiziaria di Angelelli, accettando sostanzialmente la versione ufficiale dell'incidente automobilistico. In tal senso sono rivelatrici le dichiarazioni dell'ex Presidente Videla rilasciate nel corso del processo per la morte di Angelelli, a proposito di una riunione avuta con l'allora Nunzio apostolico Pío Laghi:

Luego de ocurrido el accidente de Monseñor Angelelli, y acallados los comentarios de todo tipo a que dio lugar, en fecha que no puedo precisar; recibí en audiencia - a su pedido - al nuncio apostólico Pío Laghi. Terminado de exponer el tema que motivara la audiencia, antes que el señor Nuncio se retirara, le manifesté fuera de la agenda mi preocupación por el accidente que sufrió Monseñor Angelelli y fundamentalmente, por la posibilidad que el mismo pudiera afectar la relación entre el Gobierno y la Iglesia. El Sr. Nuncio, sin hesitar me respondió: "presidente, la Iglesia tiene asumido que el fallecimiento de Monseñor Angelelli, fue producto de un accidente; Ud. puede dormir tranquilo respecto de este asunto35.

Si tratta, naturalmente, delle dichiarazioni di uno degli imputati per la morte del vescovo, il quale aveva tutto l'interesse a cercare di dimostrare una convergenza tra le autorità militari e quelle ecclesiastiche sulla versione dell'incidente automobilistico.

34 Ibidem, p. 33.

35 Ibidem, p. 397. 
Tuttavia, la reticenza dei vertici episcopali argentini dinanzi alle indagini sulla repressione durante il periodo della dittatura militare si mostra a più riprese al momento del ritorno alla democrazia. Nell'agosto 1984 il Cardinale Aramburu, interrogato sulla possibilità di un omicidio di Angelelli, dichiarò alla rivista «La Gazeta»: «Eso hay que probarlo. Yo no tengo ningún argumento en ese sentido [...] De las averiguaciones que se hicieron ninguna daba posibilidades de que tuviera podido ser eso que se rumorea»36.

Tale attitudine non si limitava al caso di Mons. Angelelli, essa rientrava piuttosto in una visione più ampia, volta a favorire la riconciliazione piuttosto che la ricerca della verità, ponendo enfasi sul concetto del perdono. Tale impostazione veniva esplicitata nel 1987 dal Cardinale Primatesta nel documento Reflexiones para la cuaresma en la visita del Papa nel quale il vescovo di Cordoba scriveva:

Nuestros egoísmos y nuestras pretendidas justicias nos erigen en jueces de los demás, sin mirarnos en nuestros propios errores... el Papa nos dejó un día un ejemplo perdonando y visitando a su agresor [...] Los obispos nos sugieren un camino: apresurémonos a mostrar gestos de reconciliación: perdonemos con generosidad las ofensas que nos hayan hecho - todas, cualesquiera sean postremo arrepentimiento y pidamos perdón por lo que hicimos o dejamos de hacer nuestro37.

Interrogato dalla stampa in quei giorni sulla morte dei sacerdoti Longueville e Murias, Mons. Primatesta dichiarava:

Ha habido personas en la Iglesia que murieron; quién, cuándo y cómo, no me atrevo a hacer un juicio, [e aggiunse] ¿Y qué tiene que hacer la Iglesia? Tiene que rezar el padrenuestro, perdonar y nada más, y seguir adelante ${ }^{3}$.

L'atteggiamento reticente delle autorità ecclesiastiche dinanzi ai giudici che indagavano sulla morte di Mons. Angelelli sembra rafforzare la tesi di un "silenzio complice" della maggioranza dell'episcopato, denunciato da diversi testimoni e formalizzato dai giudici argentini nelle conclusioni della sentenza del Settembre 2014:

Las internas entre los distintos miembros de la Iglesia en relación a las denuncias de Angelelli y el silencio ante sus reclamos, favorecieron la decisión y oportunidad

${ }^{36}$ BARONETTO, Luis, op. cit. p.230.

37 Ibidem.

38 Ibidem. 
para cumplir con el Plan del Ejército y "aniquilar" al Obispo; ejecutando un plan perfectamente diseñado para ocultar el homicidio bajo el manto del "accidente" 39.

È solo nel 1996 che nel documento Caminando Hacia el Tercer Milenio la Chiesa cattolica argentina riconoscerà per la prima volta pubblicamente il coinvolgimento di alcuni dei suoi membri nella violenza che caratterizzò gli anni della dittatura, chiedendo perdono per i crimini commessi sia tra le fila della guerriglia che nell'apparato dello Stato ${ }^{40}$. Un ulteriore passo avanti arriverà nel settembre del 2000 quando, nel corso di una celebrazione eucaristica nella città di Cordoba, il Presidente della CEA Mons. Karlic chiese perdono:

\footnotetext{
Por los silencios responsables y por la participación efectiva de muchos de sus hijos en tanto desencuentro político, en el atropello a las libertades, en la tortura y la delación; en la persecución política y la intransigencia ideológica, en las luchas y las guerras; y la muerte absurda que ensangrentaron nuestro país ${ }^{41}$.
}

Sulla scia di tale apertura, nel 2005 l'Arcivescovo di Buenos Aires Mario Bergoglio incaricava l'Arcivescovo Carmelo Giaquinta di raccogliere le prove utili allo svolgimento del processo per l'assassinio di Mons. Angelelli, mentre la diocesi de La Rioja si costituiva per la prima volta come parte civile. Nel 2011, divenuto Papa col nome di Francisco, sarà lo stesso Bergoglio ad inviare dal Vaticano importanti documenti provenienti dalla nunziatura apostolica di Buenos Aires ${ }^{42}$.

Alla base dell'ultimo processo per l'omicidio del vescovo Angelelli - iniziato nel Novembre 2013 - vi è la valutazione che ha portato i giudici argentini a considerare l'omicidio di Mons. Angelelli come caso di Lesa Humanidad poiché «inserito in un piano sistematico di repressione portato avanti dallo Stato, con il dichiarato proposito di reprimere la sovversione»43. Di fatto però, il Gen. Menendez e il Vicecomodoro Estrella - unici accusati a causa della morte degli altri militari coinvolti nell'omicidio -

39 TRIBUNAL ORAL FEDERAL DE LA RIOJA, op. cit. p. 551.

40 «La Iglesia argentina pide perdón por su implicación en la represión militar» in El Pais.com URL: < http://elpais.com/diario/1996/04/28/internacional/830642408_850215.html > [consultato il 13 agosto 2015].

41 ROUILLON, Jorge, «Histórico pedido de perdón de la Iglesia argentina», in La Nacion.com.ar, URL: < http://www.lanacion.com.ar/32127-historico-pedido-de-perdon-de-laiglesia-argentina $>$ [consultato il 13 agosto 2015].

42 BULLENTINI, Ailin, "Cuando se callaron las Iglesias», in Pagina12.com.ar URL: < http://www.pagina12.com.ar/diario/elpais/1-249124-2014-06-22.html > [consultato il 13 agosto 2015].

43 TRIBUNAL ORAL FEDERAL DE LA RIOJA, op. cit., p. 582. 
non ebbero un ruolo diretto nell'assassinio del vescovo. Alla base della loro accusa vi è piuttosto la loro posizione all'interno della gerarchia militare. Nello specifico:

Luis Fernando Estrella desarrolló tareas propias de la lucha antisubversiva, tales como impartir órdenes a sus subalternos policiales y militares para realizar tareas de inteligencia y controlar la actividad de la Pastoral Diocesana. En el hecho que se juzga, su aporte consistió en retransmitir órdenes para reunir información de inteligencia en relación a las investigaciones llevadas a cabo por el Obispo Angelelli, como así también de sus horarios e intenciones de comunicar la información recabada a sus superiores; luego ordenó liberar la zona de controles para facilitar la comisión del hecho a los autores materiales que actuaban bajo su dominio operacional; órdenes que había recibido a través de las autoridades del Área 3.1.4, quienes, a su vez, las habían recibido de la máxima autoridad de la Zona 3; esto es, del acusado Luciano Benjamín Menéndez44.

Il processo per l'omicidio di Mons. Angelelli si chiude il settembre 2014 con la condanna all'ergastolo per il Generale Luciano Benjamin Menéndez e per il Viceammiraglio Luis Fernando Estrella quali responsabili del crimine, in virtù della loro posizione di comando all'interno dell'istituzione che pianificò e portò a termine l’omicidio.

\section{Conclusioni}

Al di là della sua rilevanza sul piano politico e sociale argentino, il "Caso Angelelli" assume un'importanza fondamentale da un punto di vista storiografico. Oltre a raccogliere le testimonianze rilasciate nel corso degli anni, l'ultimo processo ha potuto avvalersi di una serie di documenti prima inaccessibili, quali gli archivi della DIPBA, i documenti della Nunziatura inviati dal Vaticano e quelli raccolti dalla CEA. Tali elementi, oltre a chiarire il movente immediato dell'omicidio - la volontà di occultare delle prove raccolte da Angelelli sulla morte dei sacerdoti Longueville e Murias - hanno aiutato i giudici a ricostruire con maggior precisione il contesto che portò all'assassinio di Mons. Angelelli per mano di agenti del regime militare, nonché ad accendere un faro sulla questione delle relazioni tra la Chiesa cattolica e il regime militare. I documenti della DIPBA in particolare mostrano la grande attenzione riservata dai militari ai membri del clero, evidenziando come il fattore religioso fosse ritenuto centrale all'interno del PRN. Il procedimento giudiziario ha poi evidenziato lo stretto legame tra

44 TRIBUNAL ORAL FEDERAL DE LA RIOJA, op. cit., pp. 555-556. 
le autorità militari e i potentati locali della provincia de La Rioja, entrambi danneggiati nei loro intenti politici ed economici dall'azione e dalla predica del vescovo.

La corrispondenza che Mons. Angelelli intrattiene con le autorità della CEA e con il Nunzio Apostolico mostrano poi il suo isolamento all'interno delle istituzioni ecclesiastiche. Ripiegato sull'esigenza dell'unità del mondo cattolico e spaventato dalle derive oltranziste da parte del clero argentino, l'episcopato non fu mai in grado di assumere un atteggiamento di denuncia profetica nei confronti della repressione. Ciò era dovuto in parte alla solidarietà di parte dell'episcopato con il PRN, ma le ragioni di tale immobilismo vanno cercate più in profondità: nello storico legame che lo univa alle Forze Armate; nella paura di legittimare in tal modo movimenti cristiani rivoluzionari; in definitiva, nel suo eccessivo coinvolgimento allinterno del conflitto politico e sociale argentino, tale da impedirgli di ergersi al di sopra di esso e di guidare la nazione verso un'uscita pacifica dalla dittatura.

La lunga e travagliata storia processuale del caso Angelelli rappresenta infine un esempio delle difficoltà ancora presenti sulla strada del consolidamento democratico argentino. Il desiderio di verità e giustizia emerso con forza dalle indagini della CONADEP e dal giudizio alla Giunta Militare all’indomani del ritorno alla democrazia fu ben presto accantonato dinanzi alle esigenze di stabilità politica del paese. Sarà solo con l'ingresso nel nuovo millennio che il cambio politico e generazionale permetterà alla società argentina di riaprire i conti con il proprio passato, avviando una nuova stagione di procedimenti giudiziari che negli ultimi anni stanno arrivando a conclusione in un processo non esente da clamori e polemiche 45 . Lo stesso cambio generazionale è servito poi a favorire una maggiore collaborazione da parte delle autorità ecclesiastiche, i cui archivi molto hanno ancora da raccontare su uno dei periodi più drammatici della storia argentina.

45 PIKIELNY, Astrid, «Claudia Hilb: "No es razonable creer que pueda haber un solo relato sobre un pasado tan complejo"», in La Nacion.com.ar URL: < http://www.lanacion.com.ar/1619669-claudia-hilb-no-es-razonable-creer-que-pueda-haber-unsolo-relato-sobre-un-pasado-tan-comple $>$ [consultato il 13 agosto 2015]. 


\section{* L'autore}

Mirko Giancola è dottorando presso l' EHESS di Parigi. Nel 2011 consegue la Laurea Magistrale in Scienze Storiche nell'ambito del progetto di doppio diploma tra l'Università di Bologna e l'Università Paris Diderot di Parigi, con una tesi sulla circolazione delle idee in campo militare tra Europa e Sud America nella seconda metà del Novecento. Attualmente sta preparando una tesi sulle relazioni tra la Chiesa cattolica e i regimi militari in Argentina e Cile negli anni Settanta del Novecento in cotutela con l'Università Roma Tre.

URL: < http://www.studistorici.com/progett/autori/\#Giancola >

\section{Per citare questo articolo:}

GIANCOLA, Mirko, «Un passato che non passa. L'Argentina dinanzi al caso Angelelli», Diacronie. Studi di Storia Contemporanea : Le dittature militari: fisionomia ed eredità politica, 29/12/2015,

URL:< http://www.studistorici.com/2015/12/29/giancola_numero_24/ >

\section{Diacronie Studi di Storia Contemporanea $\widehat{B}$ www.diacronie.it}

Risorsa digitale indipendente a carattere storiografico. Uscita trimestrale. redazione.diacronie@hotmail.it

Comitato di redazione: Jacopo Bassi - Luca Bufarale - Elisa Grandi - Antonio César Moreno Cantano - Deborah Paci - Fausto Pietrancosta - Alessandro Salvador - Matteo Tomasoni - Luca Zuccolo

Diritti: gli articoli di Diacronie. Studi di Storia Contemporanea sono pubblicati sotto licenza Creative Commons 3.0. Possono essere riprodotti e modificati a patto di indicare eventuali modifiche dei contenuti, di riconoscere la paternità dell'opera e di condividerla allo stesso modo. La citazione di estratti è comunque sempre autorizzata, nei limiti previsti dalla legge. 